\title{
Sensitivity Analysis on the Assumption of Geometric Batch Arrivals for Real-time versus Non-real-time scheduling in a statistical Multiplexer
}

\author{
Robert A. Lackman \\ Computer Science \\ Department, Ocean \\ University of Qing Dao \\ Qing Dao 266003 PROC \\ lackman@ouqd.edu.cn
}

\author{
David Tipper \\ Department of Information \\ Sciences and \\ Telecommunications \\ University of Pittsburgh, \\ 135 N. Bellefield Ave. \\ Pittsburgh, PA 15260 USA \\ tipper@tele.pitt.edu
}

\author{
John D. Spragins \\ Retired from \\ Department of Electrical \\ and Computer Engineering \\ Clemson University \\ Clemson, SC USA \\ john.spragins@ces.clemson \\ .$e d u$
}

\begin{abstract}
Sensitivity analysis is done on the assumption of geometric batch arrivals used by several authors in the study of tradeoffs between real-time and non-real-time traffic in $a$ statistical multiplexer. This work finds the geometric batch arrival assumption conservative compared with correlated MMPP's. The Poisson arrival assumption is much more optimistic that the geometric batch arrival assumption.
\end{abstract}

\section{Introduction}

In ATM networks, a key issue is how to trade-off real-time performance versus non-real-time performance. Several ATM classes are defined in the ATM 4.0 traffic management 4.0 specification [1]. ATM defined classes that require real-time service include real-time traffic include RT-VBR traffic, while non-real-time classes of traffic include $A B R$ and UBR traffic. RT-VBR traffic include compressed video and audio. ABR and UBR include TCP/IP traffic, file transfer, email, and telnet services. Scheduling at the ATM switch is one technique that may be used to control the performance tradeoff between different classes of traffic $[2,3]$.

Several works have done analytical analysis of the performance of a statistical multiplexer where one class of traffic is real-time traffic and the other class of traffic is non-real-time.[4-8]. For real-time traffic each packet has a deadline and will be dropped if its deadline is exceeded before it is served. The performance criterion is the fraction of real-time packets that are dropped. For nonreal-time traffic there is no deadline and the performance criterion is minimal delay. These works include Chipalkatti, Kurose, and Towsley[4], Lackman, Spragins, and Tipper [5], Pingalli, and Kurose[7], Huang and Alberto Leon Garcia[8]. Others authors such as Lazar and Pacifica [9] have studied similar problems using discrete event simulation assuming Markov-Modulated Poisson Process (MMPP) arrival statistics. The authors of the analytical studies have made the assumption that the number of arrivals of real-time and non-real-time classes arriving in each constant service time are geometrically distributed. This assumption of geometrically distributed batches does not have a physical interpretation in a statistical multiplexer. A Bernoulli process would be a much more realistic assumption. See Perros and Elsayeed [10]. Rather the geometric batch assumption is made to allow an analytical analysis of the problem. It is a required assumption that is a necessary and sufficient condition for there to be a Markov chain when there is real-time traffic $[5,6]$. In this paper discrete event simulation is used to investigate how sensitive the performance is to the geometric batch arrival assumption. . Several authors have used Markov Modulated Poisson Processes (MMPP) to represent correlated arrivals. A MMPP is a nonstationary Poisson Process with an arrival rate modulated by a Markov chain [11, 12] It is shown in this paper that the assumption of geometrically distributed batches of arrivals gives results conservative compared to correlated MMPP arrivals.

In [4] the authors compare the performance of heuristic scheduling algorithms they called Minimum Laxity Threshold (MLT) and Queue Length Threshold (QLT). In MLT non-real-time is given priority until there is a real-time packet within a threshold of its deadline. In QLT real-time is given priority until the non-real-time queue length exceeds a threshold. In [4] for the parameters 
looked at it is shown that MLT and QLT give a similar trade-off and therefore since QLT is simpler to implement, it is preferred. In $[5,6]$ it is shown that while true for the parameters looked at in [4], this is not generally true. For larger real-time lifetimes, MLT becomes greatly superior to QLT in providing a trade-off between real-time and non-real-time performance.

\section{Methodology}

In $[4,5,6]$ a statistical multiplexer is studied with the traffic classes of real-time and non-real-time. The service time is a constant. Each real-time packet has an identical lifetime $\tau$. After the packet has been in the service queue for its lifetime; the packet will be discarded. For [4] stationary performance is studied. For $[5,6]$ the same problem is studied for nonstationary burst arrivals. For the bursting arrival class the arrival rate for each class is $40 \%$ of the service rate for 75 service time units, then it increases to $60 \%$ of the service rate for 50 service time units before returning to $40 \%$ of the service rate for another 125 time units. For the other arrival class the arrival rate remains at a constant $40 \%$ of the service rate. Because the analysis in $[5,6]$ is a nonstationary analysis, the non-real-time performance measure is non-real-time queue length because delay cannot be obtained from queue length for a nonstationary analysis. For real-time traffic the performance measures are real-time loss rate and real-time service rate. For the simulation results for real-time only real-time service rate is available because real-time loss rate would take excessive amounts of model execution time. But real-time service rate is an indirect indicator of real-time loss rate. Higher real-time service rate will result in lower real-time losses. The analytic techniques used in $[5,6]$ are extensions of those used in [4] to a nonstationary analysis. The simulation technique used for nonstationary analysis is to take the ensemble average at separate points in time over independent replications. [13]. For simulation results $95 \%$ confidence intervals are calculated and found to give reasonable relative processions. The largest relative precision is $2 \%$. For simplicity of viewing the confidence intervals are omitted from the graphs.

For the sensitivity analysis in this paper only simulation is a used because analytical results are not available for Poisson and MMPP arrivals.. For the simulation studies, geometric batch, Poisson and two MMPPs are compared. For both MMPPS the arrival rate transitions between .3 and .5 for the nonbursting state while it transitions between .3 and .9 for the bursting state. For $\mathrm{MMPP}_{2}$ the transition time between states averages 2 service time units while for $\mathrm{MMPP}_{5}$ it averages 5 service time units.
For the sensitivity analysis the real -time lifetime $\tau$ is 6 service time units. For all results in this paper, the MLT threshold is 4, and the QLT threshold is 9.

\section{Results}

Figures 1 and 2 give the results for a non-real-time burst with Minimum laxity threshold scheduling. The results show that geometric arrivals give the highest nonreal-time queue length and Poisson arrivals the lowest queue length with the MMPP results in between. Geometric arrivals give the lowest real-time service rate and Poisson arrivals the highest with the MMPP results in between. Figures 3 and 4 show the same characterization for non-real-time burst QLT results. Both MLT and QLT non-real-time bursts show Poisson arrivals giving the best real-time and non-real-time performance with geometric arrivals giving the worst with MMPP results in between. Space constrains prevent the inclusion of the data but similarly both MLT and QLT real-time bursts show Poisson arrivals giving the best real-time and non-realtime performance with geometric arrivals giving the worst with MMPP results in between. This demonstrates that the geometric batch arrival assumption gives fairly conservative performance results.

\section{Conclusions}

This paper gives sensitivity analysis on the geometric batch arrival assumption used by several authors in order to derive analytical solutions to explore the performance of different network scheduling algorithms. It is found that the geometric batch arrival assumption gives conservative results compared with correlated MMPP arrivals. Poisson arrivals give much more optimistic results.

\section{Bibliography}

[1] The ATM Forum TC, "Traffic Management Specification Version 4.0",aftm-0056.000, Apr. 96

[2] Ghani N., Nananukul S., Dixit Suhir, ATM Traffic Management Considerations for Facilitating Broadband Access, IEEE Communications Magazine, November 1998

[3] Aras C., Kurose J., Reeves D., Schulzrinne H, Real-time Communications in Packet Switched Networks, Proceedings of the IEEE, Vol. 82, No. 1, 1994

[4]R. Chipalkatti, J. F. Kurose, D. Towsley, Scheduling policies for real-time and Non-real-time traffic in a statistical multiplexor, Proceedings 1989 IEEE INFOCOM, Ottawa, Canada (April 1989.)

[5]Lackman R. A., Spragins, J. D., Tipper David, Scheduling real-time and Non-real-time traffic under nonstationary conditions, Annals of Operations Research 36(1992) 193-224, J. C. Baltzer, A. G. Scientific Publishing, 
[6]Lackman R.A. Nonstationary Analysis of Scheduling Bursty Real-time and Non-Real-time Traffic, Ph.D. Dissertation, Clemson University, May 1992, Clemson, South Carolina, USA [7]Pingali Sridhar, Kurose, James, F. IEEE Global Telecommunications Conference and Exhibition, volume 1 , 1991

[8]Huang Chun-Chong; Leon-Garcia Alberto, Separation Principle of Dynamic Transmission and Enqueueing Priorities for Real- and Nonreal-Time Traffic in ATM Multiplexers, IEEE/ACM transactions on Networking, Vol. 2, no 6., December 1994

[9]Hyman J. M., Lazar A. A., Pacifici G., A separation principle between scheduling and admission control for broadband switching. IEEE Joumal on selected areas in Communications, May 1993, p605-16.

[10] Perros H. and Elsayed K, Call Admission Control Schemes:: A Review, IEEE Communications Magazine, November 1996 [11] Heffes Lucantoni, "A Markov Modulated Characterization of Packetized Voice and Data Traffic and Related Statistical Multiplexor Performance, IEEE Journal on Selected Areas in Communications, pp. 856-868 Vol. SAC-4 No. 6, September 1986

"Modeling Issues in an ATM Multiplexor within a Bursty Traffic Environment, IEEE INFOCOM 1991, pp. 83-91, April 9-11, 1991

[13]Lovegrove W., Hammond J., Tipper D., Simulation Methods for Studying Nonstationary Behavior of Computer Networks IEEE Journal on Selected Areas in Communications, December 1990.

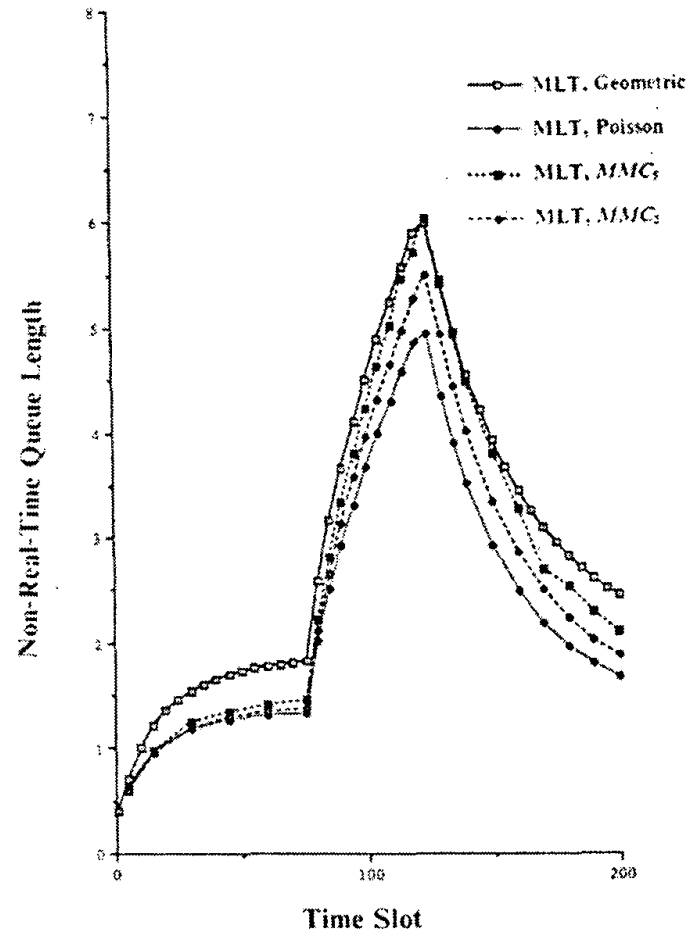

Figure-1-MLT comparison of Geometric, MMPP, and Poisson Arrivals, Non-real-time burst, Non-real-time queue length 


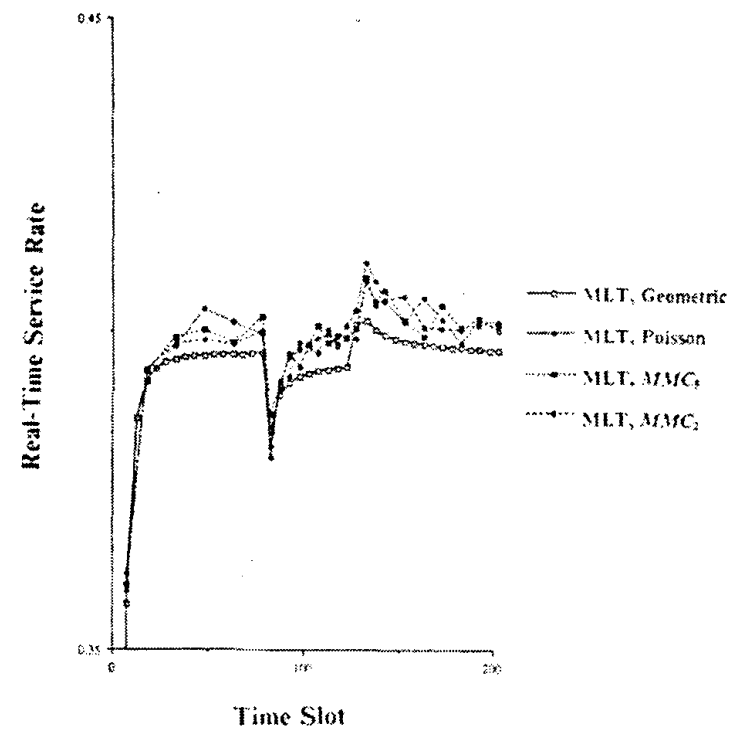

Figure -2 -MLT comparison of Geometric, MMPP, and Poisson Arrivals, Non-real-time burst, Real-timeservice Rate

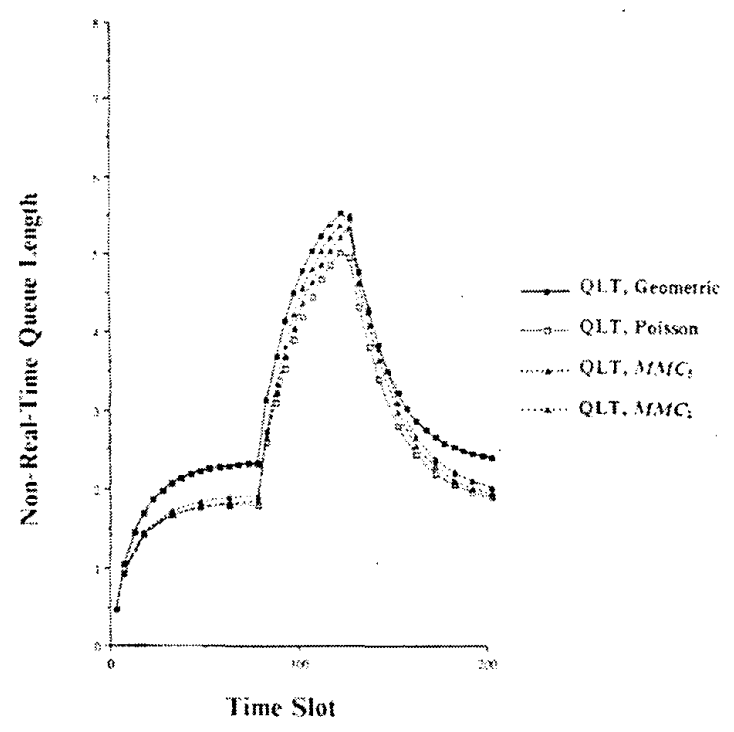

Figure 3-QLT comparison of Geometric, MMPP, and Poisson Arrivals, Non-real-time burst, Non-real-time queue length

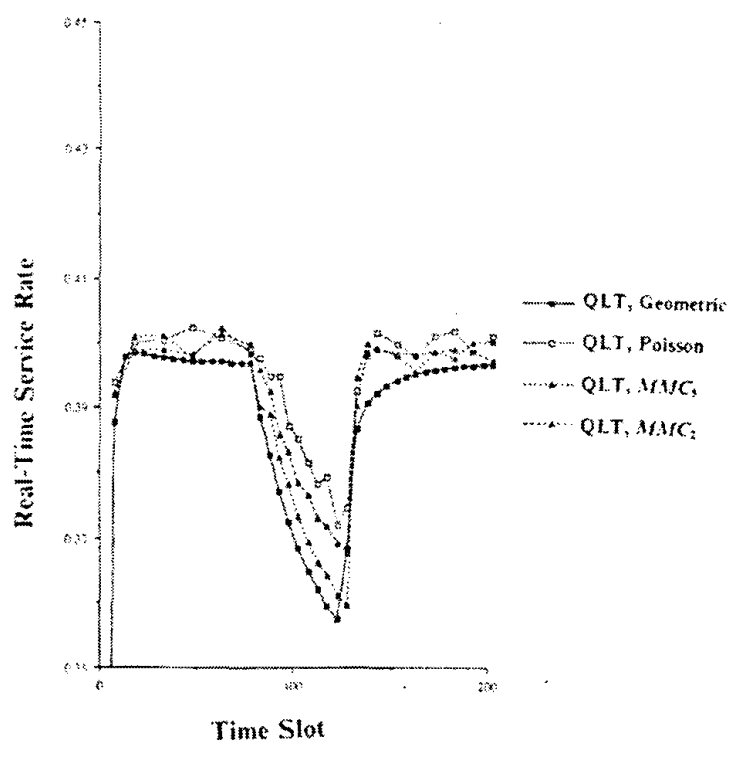

Figure 4-QLT comparison of Geometric, MMPP, and Poisson Arrivals, Non-real-time burst, Real-timeservice Rate 